\title{
Research on Adaptive Prediction Algorithm
}

\author{
Xiaoxuan $\mathrm{Ma}^{1}$ and Ya Deng ${ }^{2}$ \\ ${ }^{1}$ School of Electrical and Information Engineering, Beijing University of Civil \\ Engineering and Architecture, Beijing 10044, China \\ ${ }^{2}$ School of Electrical and Information Engineering, Beijing University of Civil \\ Engineering and Architecture, Beijing 10044, China \\ Imaxiaoxuan@bucea.edu.cn,gaga6306@126.com
}

\begin{abstract}
The paper aims to solve the problem that the nonlinear model of control system being hard to fetch parsing and impossible to be completely accurately described by mathematical model, and thus adopting adaptive predictive control algorithm for its capability of on-line identifying the variation characteristics of uncertain parameters. At the beginning, we analyze the advantages and disadvantages of many kinds of adaptive control method. Then the characteristics of cascade control system are put forward. According to the principle, we study adaptation prediction open-loop control by simulation. Later, taken interference's effect on main variable into account, we design adaptive prediction closed loop scheme to improve the system's adaptive capacity at the time the load changing, and then identify a cascade control program whose inner loop is PID controller and outer loop is self-adaptation predictive controller. At last, we analyze the simulation results and make a conclusion that closed loop self-adaptation predictive control improves stability and dynamic property when the system is under interference.
\end{abstract}

Keywords: Self-adaptation control, Cascade Control, Prediction Control

\section{Introduction}

Self-adaptive prediction control is also a control method based on mathematics model, the same as common feedback control or optimum control. The difference lies in that self-adaptation control needs less prior knowledge about model and disturbance. It needs constantly fetching information of relative model to gradually improve the model. To be specific, to process the continually identify model parameter is called the system's on-line identification. As the production process continuously going on, the model becomes more and more accurate and approaches the reality by using on-line identification. Now that the model is gradually improving, the control function based on the model is likely to gradually improve along with. Under this significance, for instance, characteristic of some certain control objects may change in the operation process, but the system can gradually adapt the kind of changing by on-line identification and controller parameters changing [1, $2,14,15]$.

The research on prediction control theory is becoming more complete, but for non-linear system, a systematic study method hasn't formed at present, for the complicacy of establishing prediction model and lacking efficient mathematic analysis tool. The frequently-used method is the approximate linearization method [3]. When a system is working in a large scale, it will unavoidably bring some model error and cause the degradation or instability of the performance of the controlled system.

The paper aims at the non-linear problem of three-tank water tank, combines prediction control strategy and gradient algorithm of parameter estimation organically, taking simulation to study the effectiveness of algorithm [13]. Self-adaptation open loop control is given out in this paper and furthermore taken the influence the disturbance on the main 
variables into account, the thesis brings up the closed control of self-adaptation prediction, and confirms a cascade control solution of PID controller as inner loop and self-adaptation prediction controller as outer loop [4-6, 9, 13]. The results of simulation indicate that the closed loop self-adaptation prediction control system improves stability and dynamic property of the system under interference effect.

\section{Adaptive Algorithms}

In the nonlinear water tank level control system of this process control experimental system [16, 17], it's hard to get analytical solution with mathematical model of the object and it's also hard to describe its dynamic characteristics approximately. Furthermore, the parameter of actual controlled object is unknown, so that self-adaptation control algorithm is proposed.

Self-adaptation control is a kind of specific nonlinear feedback control. The process states of the system can be divided into two types: One has fast rate of change and the other has slow rate of change. And the slow rate of change can be regarded as a parameter. There are two time scales: the fast one and the slow one. The fast time scale applies to normally control the normal control and the slow time scale applies to update the parameters of the adjuster. Self-oscillating and self-adaptive system (SOAS) [18-20] has a high-gain feedback loop rounding the object with the aim of enabling output ${ }^{y}$ to follow the tracks of signal ${ }^{y_{m}}$ in a broad bandwidth ${ }^{\omega_{B}}$. Thus, bandwidth ${ }^{\omega_{B}}$ will have some change and fit itself to the value as wide as possible automatically. The high-gain of the loop is maintained by relay characteristic in the feedback loop; as a result, it may bring a limit cycle oscillation. As the system of limit cycle oscillation is always being in a motivated state, its oscillation frequency needs to be correction by a lead lag filter $[7,8]$.

Self-adaptation control solution includes SOAS, gain scheduling system, STR [9] and MRAS.

Transmission gain system is a kind of open loop compensating feedback system. It can be regarded as a feedback gain and adjusted with feed-forward compensation. It's impossible to achieve the adjustment of compensation errors because there is no closed loop system performance feedback. In actual process control, as the time constant and time-lag [10] are always inversely proportional to the productivity, productivity is usually chosen as the adjustment variable [11, 12].

Self-tuning regulator (STR): The structure of which is similar to MRAS. They both have two feedback loops. The inner ring is a common feedback loop composed of a process. Its regulator has adjustable parameters of the outer ring adjustment. And the adjustment of the outer ring is based on the feedback of process input and output. The difference is that MRAS comes from deterministic servo problem while STR comes from random adjustment problem. For the parameters setting problem of the regulator, the parameters of MRAS is directly updated while the ones of STR is updated by parameter estimation and control design calculation.

The experiment in the paper uses MIT law in MRAS solution. Its structure is shown in Figure 1:

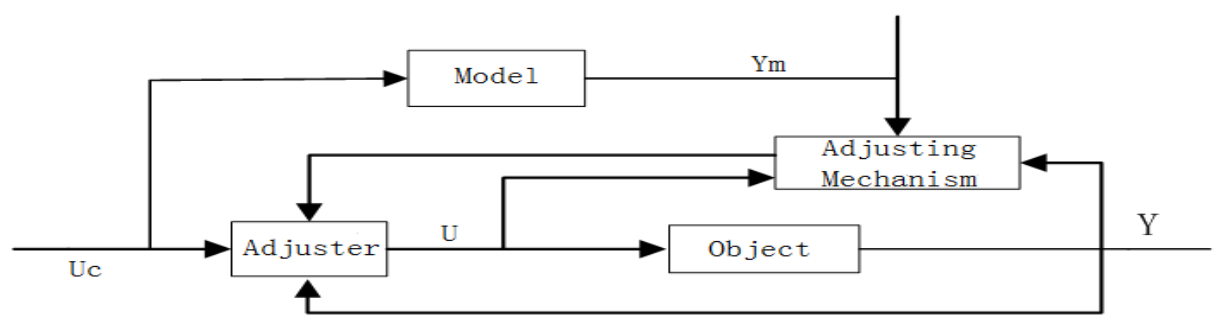

Figure 1. MRAS Structure 
MRAS is adopted to solve the control problem of performance specification fixed by reference model. The reference model indicates how the process output can response the command signal ideally. The adjuster includes two loops: The general feedback loop that composed of process and adjuster is the inner loop and the loop tuning adjuster parameters is outer loop. The outer loop tuning regulation is to minimize the error avenue between model output ${ }^{y_{m}}$ and error $e$, inside the law MIT is adopted (a parameter adjusting mechanism used in the early MRAS):

$$
\frac{d \theta}{d t}=-\gamma e \frac{\partial e}{\partial \theta}
$$

In the formula: $e$ represents error, the component of vector $\frac{\partial e}{\partial \theta}$ is the sensibility reciprocal of error relative to each adjustable parameter $\theta$. The sensibility reciprocal can be approximated by an output of linear system. Parameter $\gamma$ determines adaptive speed of the system. Specific regulation of MIT law is as follows: Suppose the changing speed of parameter $\theta$ is much slower than that of others'. In order to diminish the square of error, the most reasonable way seems to alter parameter along the negative gradient direction of $e^{2}$.

\section{Cascade Control System}

\subsection{The Conceptual Framework of Cascade Control System}

Cascade control system is double closed-loop or more closed-loop control system, the inner loop of which is sub-control object and the outer loop of which is main control object. The function of inner loop is to handle the effect of external disturbance in inner loop to avoid its fluctuation to outer loop, by this way the system's rapidity and quality are improved. Therefore, when choosing inner loop of cascade control system, one must take its response speed being far quicker than outer loop into account.

\subsection{Principle of Cascade Control System}

Two closed loops are formed in the structure of cascade control. One is in the closed loop, called inner loop, deputy loop or sub-control loop, the controller of which is vice-controller, and play a 'rough adjustment' in control process; Another is out of the closed loop, the controller of which is called main controller, and plays a 'fine adjustment' in the control process to meet the demand of controller variable at last. Take the output of the main controller as the given value of the sub-controller while use the output of sub-controller to control the controlled object. Figure 2 shows the structure of cascade control system.

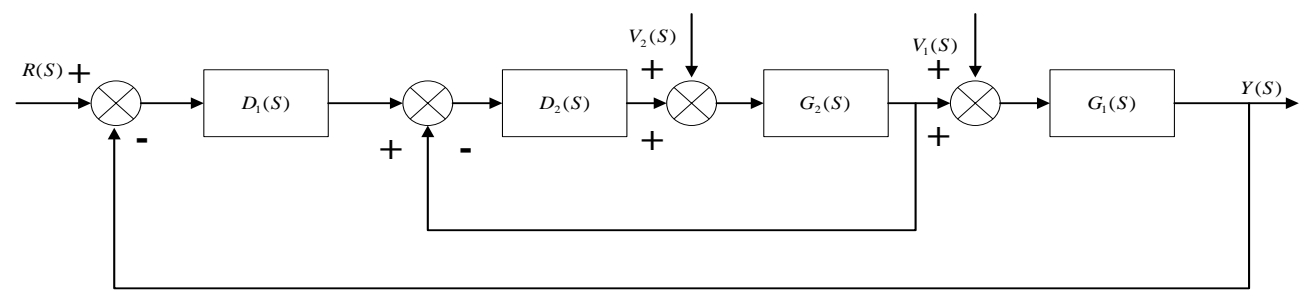

Figure 2. The Structure of Cascade Control System

\subsection{Characteristics of Cascade Control System}

(1)Sub-control loop has the characteristic of rapidity with which it can effectively overcome the secondary interference that getting into the sub-control loop. Figure 3 
shows a structure chart of simplified cascade control system, and $G_{v 2}(s)$ showed in the figure is the channel transfer function of the secondary interference.

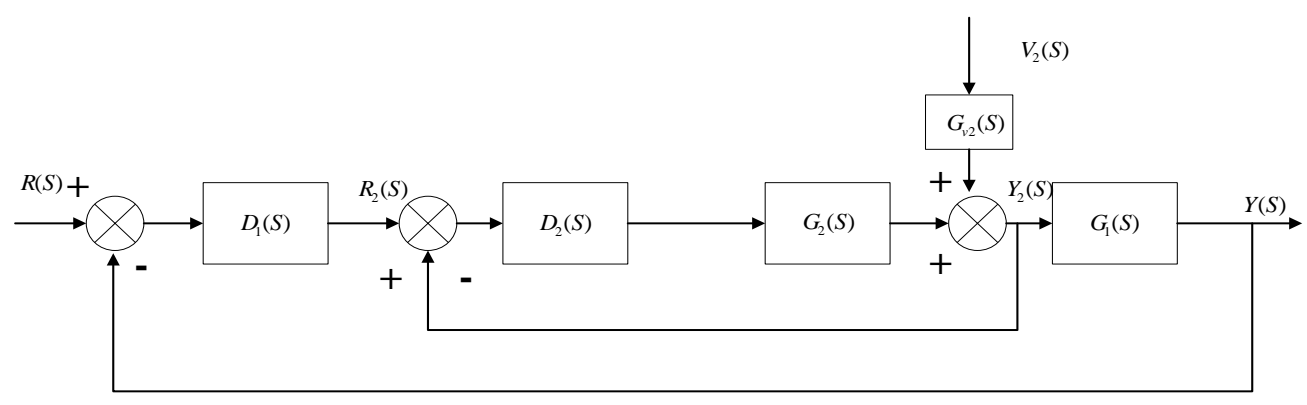

Figure 3. A Structure Chart of Simplified Cascade Control System

When the secondary interference enters the sub-control loop through the disturbance channel $G_{v_{2}}(s)$, it firstly influences the sub-paramete $Y_{2}(s)$, so the vice-controller stops immediately to weaken the interference's influence on $Y_{2}(s)$. Obviously, the interference's influence on $Y(s)$ would be reduced for entering the main control loop after restrained by vice control loop. According to the cascade system shown in Figure 3, the transfer function between the secondary interference $V_{2}(s)$ and the main parameter $Y(s)$ is:

$$
\frac{Y(s)}{V_{2}(s)}=\frac{G_{v_{2}}(s) G_{1}(S)}{1+D_{2}(S) G_{2}(S)+D_{1}(S) G_{1}(S) D_{2}(S) G_{2}(S)}
$$

In order to compare with a simple single loop system, from Figure 4 we can get the transfer function between interference $V_{2}(s)$ and the main parameter $Y(s)$ under single loop control:

$$
\frac{Y(S)}{V_{2}(S)}=\frac{G_{v 2}(S) G_{1}(S)}{1+D(S) G_{1}(S) G_{2}(s)}
$$

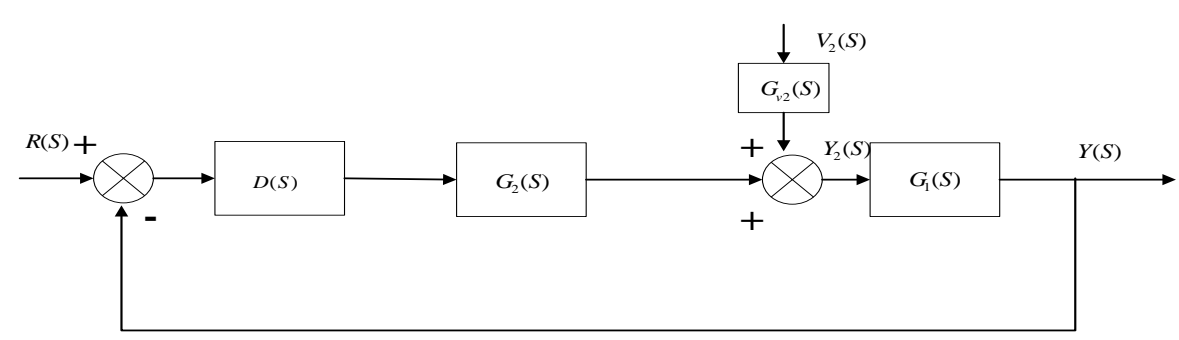

Figure 4. A Structure Chart of Single Loop Control System

Compare Figure 2 and Figure 3, if we suppose $D(S)=D_{1}(S)$, we can find that there would be one more item in the denominator of $Y(S) / V_{2}(S)$ in the cascade system. Under the working frequency of the main control loop, the coefficient of the product is generally larger and it becomes larger with the increase of the proportional gain of the vice controller. In addition, the third item in the denominator of Figure 1 has one more $D_{2}(S)$ than the second item in the denominator of Figure 2. In general, the proportional gain of the vice controller is bigger than 1 , so we can say that the structure of cascade control system makes the dynamic gain that the secondary interference $V_{2}(S)$ working on the channel of the main parameter $Y(S)$ decreases obviously. When the 
secondary interference arises it will in no time be overcome by the vice controller. Compared with single control system, the influence will reduce to $1 / 100 \sim 1 / 10$ times of their original that the secondary interference working on the controlled variable.

(2) As the vice control loop helps to improve the dynamic characteristics of the object, the system's working effective can be improved by increasing the main controller's gain. If we regard the whole vice control loop as an equivalent object, denoted as

$$
G_{2}^{\prime}(S)=\frac{Y_{2}(S)}{R_{2}(S)}
$$

At the same time, we suppose the transfer function of the vice control loop as:

$$
\begin{array}{r}
G_{2}(S)=\frac{K_{p 2}}{T_{p_{2}} S+1} \\
D_{2}(S)=K_{c 2}
\end{array}
$$

Then, the equivalent transfer function of the vice control loop will be:

$$
G_{2}^{\prime}(S)=\frac{Y_{2}(S)}{R_{2}(S)}=\frac{D_{2}(S) G_{2}(S)}{1+D_{2}(S) G_{2}(S)}=\frac{\frac{K_{c 2} K_{p 2}}{1+K_{c 2} K_{p 2}}}{1+\frac{T_{p 2} S}{1+K_{c 2} K_{p 2}}}=\frac{K_{p 2}^{\prime}}{T_{p 2}^{\prime}+1}
$$

Here

$$
K_{p 2}^{\cdot}=\frac{K_{c 2} K_{p 2}}{1+K_{c 2} K_{p 2}}
$$

And

$$
T_{p 2}^{\cdot}=\frac{T_{p 2}}{1+K_{c 2} K_{p 2}}
$$

are respectively the gain and time constant of the equivalent object.

Compare $G_{2}(S)$ and $G_{2}^{\prime}(S)$, as the in-equation $1+K_{p 2} K_{c 2}>1$ forever holds, we get $T_{p 2}^{\prime}<T_{p 2}$. The function above indicates that the existence of the vice control loop plays a role in improving dynamic characteristics, the time constant of the equivalent object reduces $1+K_{p 2} K_{c 2}$ times and it becomes larger with the increase of the proportional gain of the vice controller. In general, the proportional gain of the vice controller is suggested to get a larger one, by doing this, the equivalent time constant can decrease to a rather low numerical value, thereby, it increases the response speed of the vice control loop and also improves the working frequency of the system.

(3)The existence of the vice controller improves the adaptive ability of the system.

As we all know, productive process often contain some nonlinearity factors. Therefore, under certain load, i.e., under definite working situation, the controller parameter which set with certain controlled quantity index is only adapted to a limited extent near working point. If the load changes too much exceeding this range, the control quality will decrease. If no other solution is taken, the problem will be hard to solve in single loop control system. The situation is different in cascade system, load changing causes parameters changing in all segments, and it can play a less effect or no effect on the control quality of the system. In general, $K_{p 2} K_{c 2}>1$, so it is thus clear in function that when the gain $K_{p 2}$ of the vice controlled object changes with load, it has less influence on gain $K_{p 2}^{\prime}$ of the equivalent object. Therefore, under the circumstance of no changing the setting 
parameters of the controller, the vice controlled loop of the system will overcome the influence of the nonlinear factors and keep or approach the original controlled quality. On the other hand, the main control loop is a following control system in a cascade system. The main controller can adjust the setting value of the vice controller according to different operating conditions or load changing. The vice loop can trace rapidly to ensure the control quality of the system. From the above two aspects we can know that a cascade control system has certain adaptive ability towards load changing.

\subsection{The Design of Main controller and Sub-controller in Cascade Control}

Based on the characteristic of cascade control, in order to give full play to the function of cascade control and to improve the performance to meet the requirement of satisfaction, generally speaking, the design of cascade control system should obey the following rules:

(1) The main disturbance of system should be included in sub-control loop. The reason to take main disturbance into the sub-control loop is to greatly diminish the influence of disturbance before it affecting main controlled parameters.

(2) Try to put integration element in to sub-control loop. The lag angle of phase of integration element is $-90^{\circ}$. When an integration element is put into the sub-control loop, it can diminish the lag angle of phase, and thus benefit to improve the system's quality.

(3) It is a must to take a measurable intermediate variable to be a parameter of sub-control variables, or deduce an intermediate variable of the upstream state by the downstream state.

(4) Sampling period of the main and sub control loop is $T_{1}=(3 \sim 10) T_{2}$, thus is taken to avoid the relative interference and resonance between them.

PID control law is frequently used in the main and sub controllers. To the main controller, in order to diminish steady state error and increase control precision, an integral control is suggested; to rapid the system's response, a differential control is suggested. Thus, the main controller usually adopts PID law. For the sub-controller $D_{2}(Z)$, proportional control is generally selected and used. While on the occasion that the proportionality not permitted to be too large, integral control should be added, i.e., adopting PI control law. When PID control law is adopted, it is necessary to tuning the parameter of the controller. A cascade control is tandem connection by the two controller main-controller and sub-controller. Either parameter of them changes, the whole system will be influenced. Therefore, the tuning of cascade control system is more complex than single loop system. A kind of 'successive approximation' tuning method will be introduced below:

(1) The first step is to tuning sub control loop. Break the main control loop and get tuning parameter of sub controller in the light of single loop system tuning method, noted as $D_{1}\left(Z_{1}\right)$.

(2) The second step is to tuning main control loop. Put parameter of the sub controller on the just tuning value and close the main control loop, then regard sub control loop as an equivalent object to the main control loop, and later evaluate the tuning parameter $D_{1}\left(Z_{1}\right)$ of the main controller by single loop system tuning method.

(3) The third step is to tuning the sub control loop again. At this time, both the main and sub control loops are closed, and the system is at a cascade running status. Put the parameter of the main controller to $D_{1}\left(Z_{1}\right)$ and using single loop tuning method to evaluate the tuning parameter of the sub controller $D_{1}\left(Z_{2}\right)$. At this point, the first circulation of tuning is finished. If the control quality met the demands, the tuning task ends right then.

(4) If the control quality hasn't met the demand after the first circulation, then more 
tunings need to be taken by using above method until reach the ideal results.

\section{Simulation Study on Self-adaptation Prediction Algorithm}

\subsection{The Mathematic Model of Non-linear Object's Characteristics}

The simulation has taken the mathematic model of three-tank water level control system as reference. The schematic diagram of tank water level is shown in Figure 5. After putting inverted triangle matter in the tank, we get the mathematic model with non-linear characteristic of the system. Give the characteristic change parameter of the random process after simplifying as $v$ and the height of the tank level as $h$.

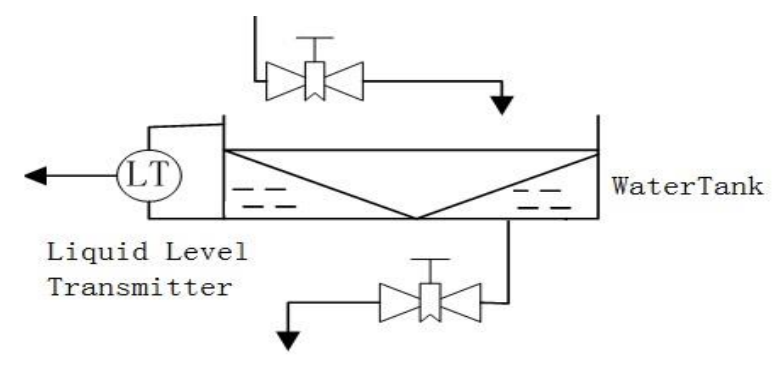

Figure 5. Schematic Diagram of Water Tank

\subsection{Open-loop Control Of Self-Adaptation Prediction Algorithmic}

Firstly, we study the open-loop control strategy of self-adaptation prediction algorithmic, and establish a simulation model in Simulink, as shown in Figure 6.

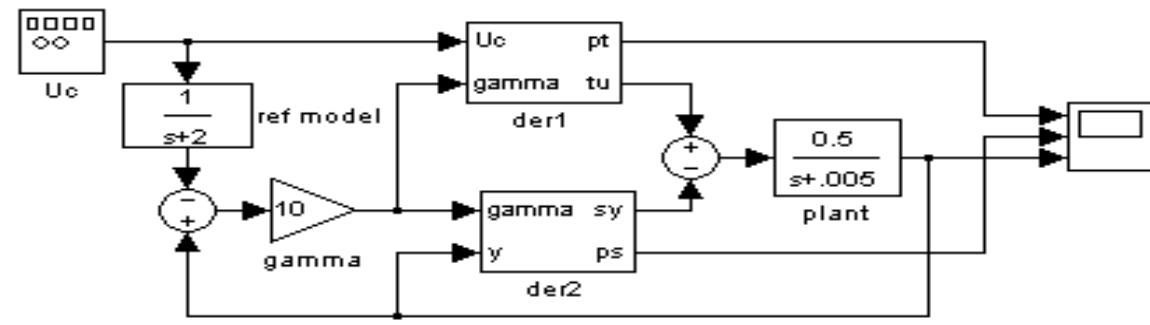

Figure 6. Simulation Model Figure

In the figure, ref model is the model, der1 is the adjusting mechanism, der2 is the adjuster and plant is the object.

The model of der1 (the adjusting mechanism) is shown in Figure 7.

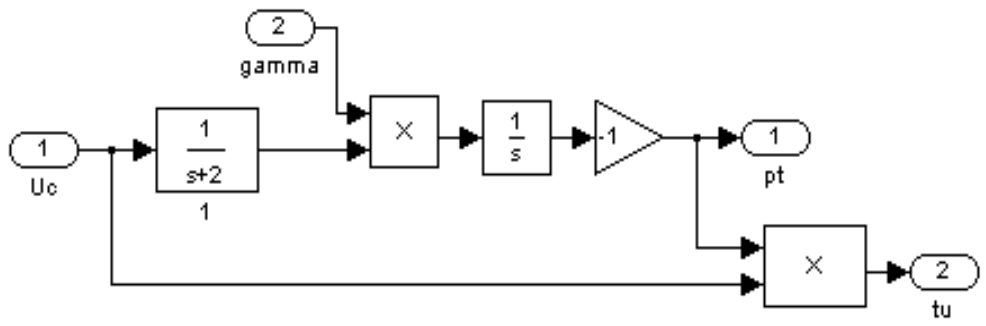

Figure 7. Adjusting Mechanism Model 
The model of der2(the adjustor) is shown in Figure 8:

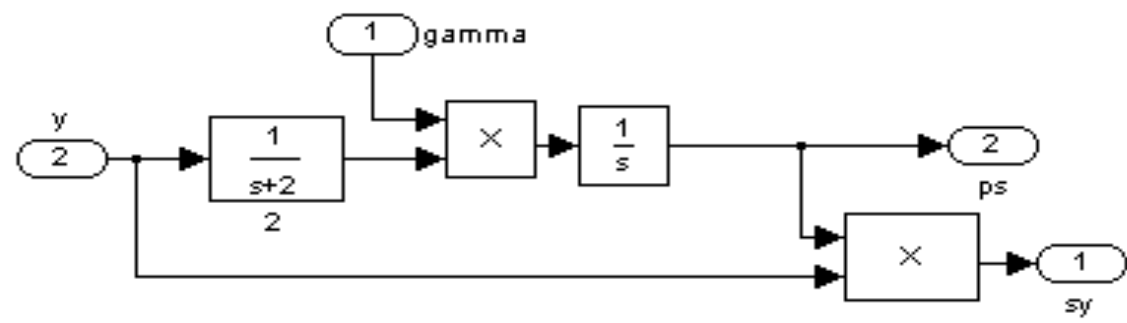

Figure 8. the Adjustor Model

The initial parameter is $\frac{1}{s+2}$, the object is $\frac{0.5}{s+0.005}$. From the variation of adaptive gain we get the parameter adjustment curve (as shown in Figure 9) and system response curve (as shown in Figure 10):
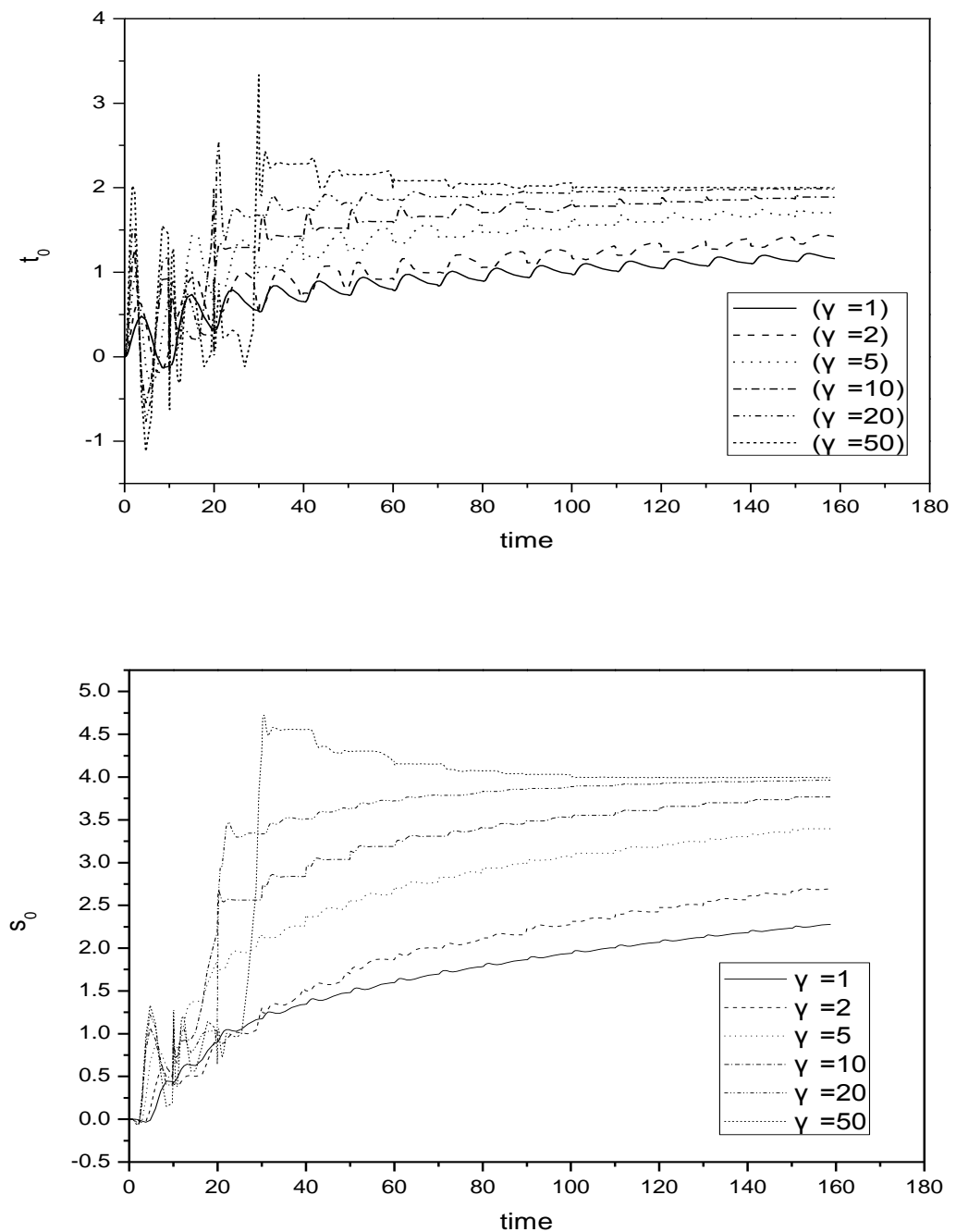

Figure 9. Parameter Adjustment Curve 


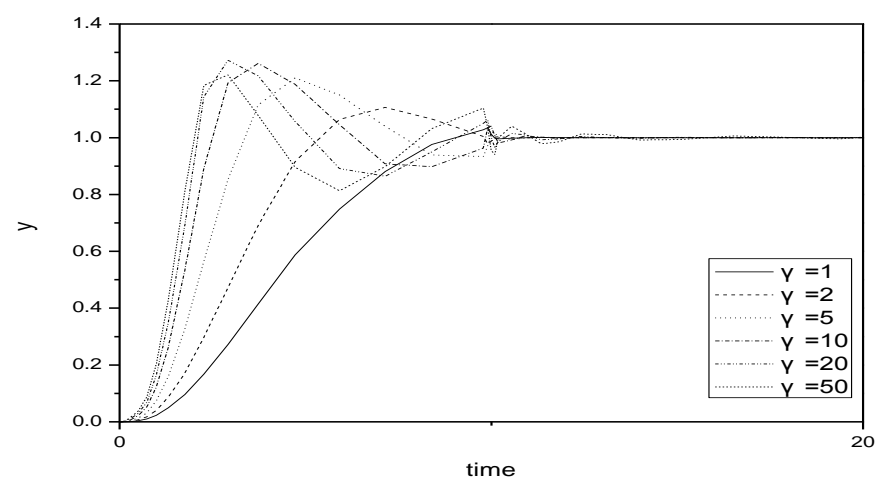

Figure 10. System Response Curve

From the above simulation we know that the speed of phase-plane trajectory of $t_{0}$ and $s_{0}$ 's approaching the actual value increasing along with the increasing of $\gamma$ within certain realms, i.e., the degree deepen with the approach of $\gamma$ in the same period. But it will bring a larger fluctuation. According to Theoretical analysis and simulation experiment the above derivation to get the verification, of which $\gamma$ is equivalent to parameter adjustment step. The long the adjustment step is, the bigger $\gamma$ will be. But a oscillation phenomenon could appear. Overall consideration, we take $\gamma=20$. Thus, overshoot is $5 \%$ and adjust time is $10 \mathrm{~s}$.

Some explanations of using MIT law to get parameter adjust law:

(1) It is not necessary to make complete model tracking, especially in non-linear system.

(2) When the error model structure appearing, the product of $\frac{\partial e}{\partial \theta}$ and $e$ is to sent to adjustor through the second multiplying unit, formula (10) and (11) are the parameter equations of adjusters. In order to get a realistic parameter adjustment law, we should use an approximation method.

$$
\begin{aligned}
& \frac{d t_{0}}{d t}=-r\left(\frac{1}{p+a_{m}} u_{c}\right) e \\
& \frac{d s_{0}}{d t}=-r\left(\frac{1}{p+a_{m}} y\right) e
\end{aligned}
$$

\subsection{The Closed Control of Self-adaptation Prediction Algorithmic}

Taking PID algorithmic as the inner controller of the whole control system and adopting self-adaptation prediction algorithmic in outer controller to form a cascade control of non-linear system, as shown in Figure 11: 


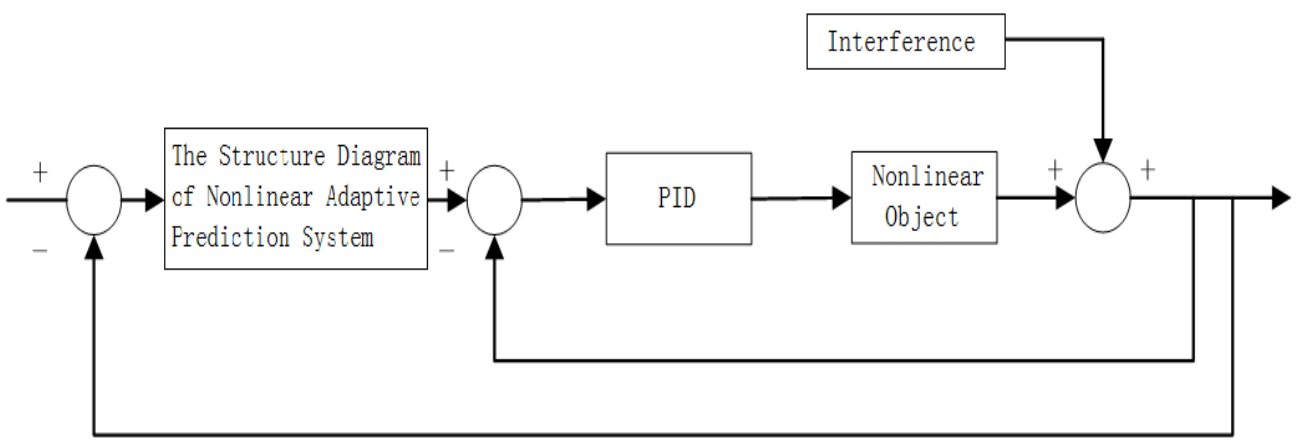

\section{Figure 11. Cascade Control Structure Schematic Diagram of Non-Linear System}

In that no system can be completely accurately described by mathematic model, we define a error prediction model:

$$
e(k)=y(k)-y_{m}(k)
$$

Here, $y(k)$ is the actual output value at $k$ moment, and $y_{m}(k)$ is the prediction output value of $k$ moment. The error $e(k)$ is composed of the actual output $y(k)$ which contains un-measurable external disturbance $\varepsilon(k)$ and the error of prediction model output $y_{m}(k)$.

The closed prediction has the same function with on-line correction. The prediction value can be given by model prediction value $y_{m}(k)$ and the weighting $\omega$ of error value in the above error model:

$$
y_{p}(k+i)=y_{m}(k+i)+\omega e(k)
$$

Combined with the self-adaptation module above, we reestablish the closed loop prediction module and get the Simulink model as shown in Figure 12:

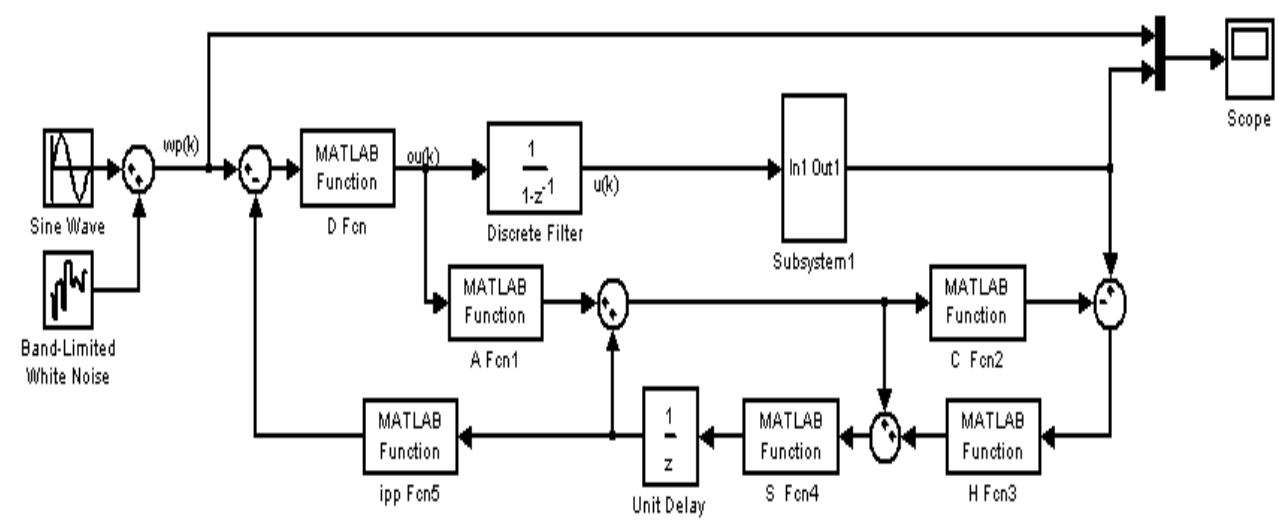

Figure 12. Closed Loop Prediction Simulation Model

By giving a sinusoidal signal as the input and a write noise signal as the disturbance, we get the prediction output response as shown by the dotted line as shown in Figure 13:

From the simulation figure 13 we can find that the predictive value has good tracking performance on the actual output value. Not only the delay is lesser, but also the adjust speed is faster. Thus, we can conclude that closed loop self-adaptation prediction control promotes the steady-state and dynamic performances under the effect of interference. Meanwhile it improves the robustness on model mismatch and environmental disturbance to a certain extent. 


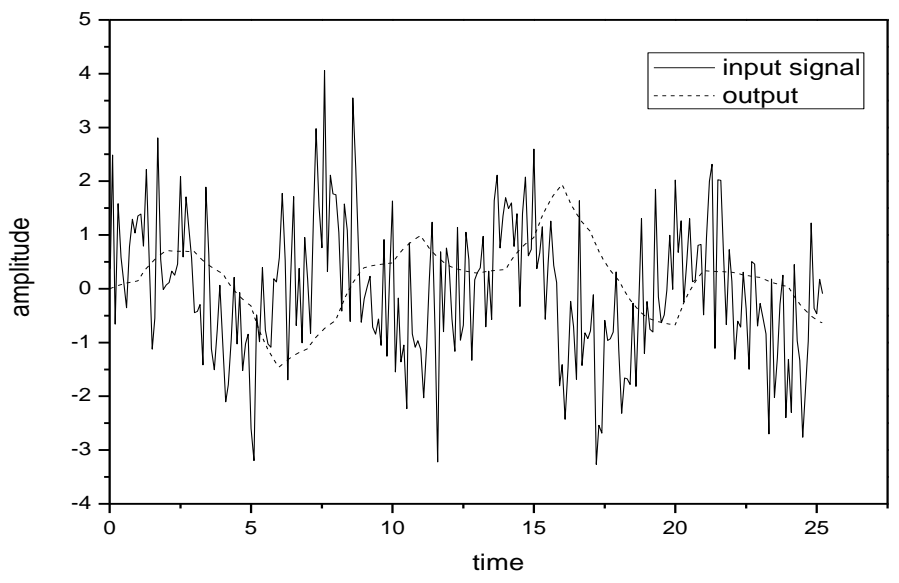

Figure 13. Prediction Output Response Diagram

\section{Conclusion}

The paper aims to solve the problem of the nonlinear model of control system being hard to fetch parsing and impossible to fully be exactly described by mathematical model, and adopting adaptive predictive control algorithm for its capability of on-line identifying the variation characteristics of uncertain parameters. At the beginning, we study self-adaptation control with simulation method and the result shows that although the unit step response of the system has good steady-state performance, but the dynamic performance changes depending on the adjustment of the adaption control parameter. And the larger the overshoot is, the longer the adjustment time will be. Then on considering the disturbance's influence on the main variables, we suggest the self-adaptation prediction closed loop control solution to improve the adaptive capacity of the system at the time load changes, and design a cascade control solution whose inner loop is PID controller and outer loop is self-adaptation predictive controller. The result of simulation indicates that with the action of disturbance, the predicted value can greatly match the variation of actual output value, in not only less delay but also faster adjust speed. Therefore, the closed loop self-adaptation prediction control has successfully promoted the steady-state and dynamic performances under the effect of interference.

\section{Acknowledgement}

This work was funded by Science and Technology Project of Ministry of Housing and Urban-rural Development of the People's Republic of China (Grant No.2014-K8-057), Beijing Municipal Outstanding Talents Fund (Grant No. 2011D005017000002), Science and Technology Project of Beijing Municipal Commission of Education (Grant No. KM201410016002).

\section{References}

[1] Q. M. Zhu, K. Warwick and J. L. Douce, "Adaptive General Predictive Controller for Nonlinear Systems", IEEE proc. Control Theory Application, no. 138, (1991), pp. 33-40.

[2] Q. M. Zhu and S. A. Billings, "Parameter Estimation for Stochastic Nonlinear Rational Models", Int. J. Control, vol. 57, no. 2, pp. 309-333.

[3] C. J. Qian and W. Lin, "A Continuous Feedback Approach to Global Strong Stabilization of Nonlinear Systems", Transaction on Automatic Control, vol. 46, no. 7, (2001), pp. 1061-1078.

[4] A. B. Miguel, J. J. Ton and D. B. Van, "Predictive control based on neural network model with I/O feedback linearization”, Int. J Control, vol. 72, no. 17, (1999), pp. 1358-1554.

[5] H. Shouting, K. Reif and R. Unbehauen, "A neural approach for control of nonlinear systems with 
feedback linearization”, IEEE Trans. On Neural networks, vol. 9, no. 6, (1998), pp. 1409-1420.

[6] F. Zhigang and W. Shifu, "Nonlinear Neural Predictive Control with Control Constraints", Proceeding of the 3rd world congress on intelligent control and automation, (2000), pp. 1081-1083.

[7] L. Xiongfei, G. Jinding and Q. Haibing, "A New Way on FPGA Implementation of LMSA daptive Filter", Piezo electectrics \& Acoustooptics, vol. 29, no. 1, (2007), pp. 87-89.

[8] L. Hongwei and Z. Changming, "Application and Comparison between FIR and IIR Digital Filter Synthesis Using LMS”, Modern Electronics Technique, no. 15, pp. 79 81.

[9] Y. Qingsong and H. Hao, "Performance Comparisons and Simulations of Variable Step Size LMS Algorithms", Shanxi Electronic Technology, (2007).

[10] T. P. Zhang and S. Ge, "Adaptive Dynamical Surface Control of Nonlinear Systems with Unknown Dead Zone in Feedback From”, Automatic, vol. 44, no. 7(2008), pp. 1895-1903.

[11] Y. Gao and Z. Zhang, "Calculation of read distance in passive backscatter RFID systems and application", Journal of System and Management Sciences, vol. 2, no. 1, (2012), pp. 40-49.

[12] Z. Xiang, W. Deshi and L. Jingxi, "The Simulation of Variable Step-Size LMS adaptive algorithm", Microcomputer Information, vol. 19, (2007), pp. 252-253.

[13] L. Maoqiong, "Based on the damped least square method of system identification and intelligent control", Tianjin, Nankai university, (1999).

[14] X. L. Wu, H. R. Jia, H. Meng, et al., "Research of the Intelligent Controls Methods and Applications on Nonlinear Process", Beijing: National Defense Industry Press, (2006), pp. 102-106.

[15] X. Y. Xu, "Adaptive Control Theory and Application", Beijing: Publishing House of Electronics Industry, (2007).

[16] F. Chunbo and F. Shumin, "Nonlinear control system analysis and design”, Beijing, Publishing House of Electronics Industry, (1998), pp. 125-127.

[17] H. K. Khalil, "Nonlinear systems", Publishing House of Electronics Industry, (2001).

[18] J. M. Tang, "Enhanced transmission of nonlinear optical loop mirrors using SOAs with robust transparency", Lasers and Electro-Optics Society 2000 Annual Meeting (LEOS), vol. 1, (2000), pp. $110-111$.

[19] L. Hsu, "Self-oscillating adaptive systems (SOAS) without limit-cycles, American Control Conference", Proceedings of the 1997, vol. 3, (1997), pp. 2155-2159.

[20] S. Li, X. Li, J. Shen and Z. Hong, "SNR of 3-stage fiber delay lines based on SOAs in microwave photonics link", Wireless and Optical Communications Conference (WOCC), 19th Annual, (2010), pp. $1-3$.

\section{Authors}

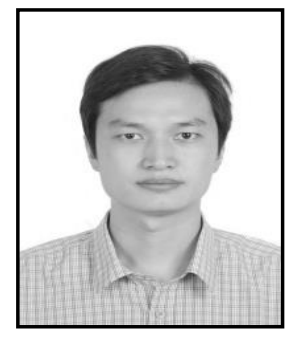

Xiaoxuan Ma, He received the $\mathrm{PhD}$ degrees from BeiHang University in 2010. Since 2010, he has been a lecturer at the School of Electrical and Information Engineering, Beijing University of Civil Engineering and Architecture. His research interests include data mining, distributed systems, distributed algorithms, adaptive prediction algorithms.

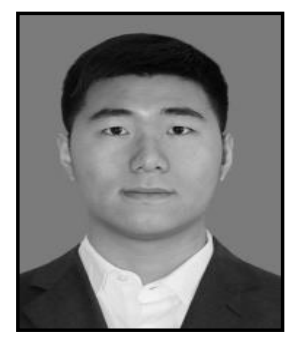

Ya Deng, He was born in 1990. Since 2013 he has been a Graduate student of School of Electrical and Information Engineering, Beijing University of Civil Engineering and Architecture. Her research interests include building electrical and intelligent, distributed systems. 